\title{
Synthesis and Characterization of $\mathrm{Au}-\mathrm{Zn}$ Nanoalloy by Laser Irradiation in Liquid Media
}

\author{
F. HAJiesmaeilbaigi ${ }^{a}$, M. FAzEli JADIDI $^{b}$ AND A. MotAmedi ${ }^{a, *}$ \\ ${ }^{a}$ Laser \& Optics Research School, NSTRI, P.O. Box 11365, 8486 Tehran, Iran \\ ${ }^{b}$ Department of Science, Faculty of Physics, University of Zanjan, Zanjan, Iran
}

\begin{abstract}
Alloy nanoparticles due to their possibility of regulation, the region of absorption peak and consequently their optical and electrical properties, have a specific significance. In this research, nanosecond laser pulses of Nd:YAG laser with $532 \mathrm{~nm}$ wavelength that is close to absorption peak of gold and zinc nanoparticles, were used for synthesis of alloy. Atomic absorption spectrum UV-Visible, X-ray diffraction analysis and transmission electron microscopy images were utilized for characterization as well. For synthesis of $\mathrm{Au}-\mathrm{Zn}$ alloy nanoparticles, zinc and gold nanoparticles were prepared separately by laser ablation method and then the mixture of their colloidal solutions with specified ratio was exposed to laser radiation. Thereby, gold and zinc nanoparticles were combined by absorbing of laser beam and gaining of required energy for melting, and $\mathrm{Au}-\mathrm{Zn}$ alloy nanoparticles were formed. Wavelength of $532 \mathrm{~nm}$ is more effective in the formation of alloy than $1064 \mathrm{~nm}$ because of being close to wavelength of surface plasmon resonance of gold and zinc nanoparticles. Increase of concentration of gold nanoparticles compared to zinc nanoparticles in the mixed solution causes that gold nanoparticles absorb the most of the energy of laser and then larger gold particles are created, so alloy nanoparticles are not formed. The best time duration for irradiation and accomplishment of alloy formation is $30 \mathrm{~min}$.
\end{abstract}

PACS: $81.16 . \mathrm{Mk}$

\section{Introduction}

During recent years, metal and alloy nanoparticles have been more considered because of their special optical and electrical properties. These properties are completely related to the size of particles which are not seen in the bulk state [1-10]. Metal nanoparticles have plasmon absorption resonance band that for different metals with different dielectric equations, their absorption peak is changed in the ultraviolet-visible region [11]. The position of plasmon resonance peak is related to the size of particles and refraction coefficient of environment. By increasing the size of particles and the refraction coefficient, the surface plasmon resonance is desired to red wavelength. Alloy nanoparticles due to their possibility of regulation the region of absorption peak and consequently their optical and electrical properties by changing in alloy combination have a specific significance [5]. $\mathrm{Au}-\mathrm{Zn}$ alloy nanoparticles have been considered by chemical methods before [12]. But in this research, $\mathrm{Au}-\mathrm{Zn}$ alloy nanoparticles have been synthesized by laser ablation method and laser irradiation. In this research, at first, gold and zinc colloidal nanoparticles were produced by laser ablation method and then a mixture of monometal colloidal solutions of gold and zinc nanoparticles have been irradiated by laser. Gold nanoparticles and zinc nanoparticles are melted by absorbing of laser beam and catching required energy and combined together and finally $\mathrm{Au}-\mathrm{Zn}$ alloy nanoparticles are formed. The wavelength of laser has main effect on the procedure of alloy synthesis because of the difference of plasmon absorption wavelength of alloy formative nanoparticles and conse-

\footnotetext{
* corresponding author
}

quently different energy absorption from laser beam [5]. In this research, nanosecond laser pulses of $\mathrm{Nd}$ :YAG laser with $532 \mathrm{~nm}$ wavelength that is close to absorption peak of gold and zinc nanoparticles, were used for synthesis of alloy. Atomic absorption spectrum UV-Visible, X-ray diffraction (XRD) analysis and transmission electron microscopy (TEM) images were utilized for consideration and characterization as well.

\section{Experimental method}

In this research, colloidal solutions of gold and zinc metal nanoparticles have been produced using laser ablation of metal plates in pure ethanol [10]. By using a pulsed active Q-switched Nd:YAG laser $(\lambda=1064 \mathrm{~nm})$ with $20 \mathrm{~ns}$ pulse width operated at $10 \mathrm{~Hz}$ repetition rate, gold and zinc metal plates with high purity (99.99\%) were separately irradiated in $10 \mathrm{~cm}^{3}$ pure ethanol. The conditions of experiments are shown in Table.

After preparing colloidal solutions of metal nanoparticles of gold and zinc separately, the mixture of them was irradiated in a quartz cell. As a result of laser irradiation of the mixture of gold and zinc colloidal solutions, without adding any surfactant, $\mathrm{Au}-\mathrm{Zn}$ alloy nanoparticles were formed. During irradiation and afterwards, the color of solution was changed in comparison with the color of the primitive mixture of gold and zinc colloidal nanoparticles and this change in the color indicates the alloy formation. The absorption spectrum of the colloid was recorded with UV-VIS-NIR spectrophotometer.

The morphology of nanoparticles was studied with the help of a TEM. For this purpose, a droplet of alloy colloidal solution was dried on a copper grid covered by a carbon layer. The component materials of structure of prepared alloy were considered by XRD spectrum. For 
this purpose, a few droplets of alloy colloidal solution were dropped on a silicon piece and allowed to dry at the environment temperature. Then XRD analysis was accomplished on the layered surface.

TABLE

The conditions of experiments for formation of $\mathrm{Au}-\mathrm{Zn}$ alloy nanoparticles in ethanol.

\begin{tabular}{c|c|c|c|c|c|c|c}
\hline \hline Sample & $F\left[\mathrm{~J} / \mathrm{cm}^{2}\right]$ & $W[\mathrm{~cm}]$ & $R[\mathrm{~Hz}]$ & $V[\mathrm{ml}]$ & $E[\mathrm{~mJ} / \mathrm{pulse}]$ & $t[\mathrm{~min}]$ & $\lambda_{\text {rad }}[\mathrm{nm}]$ \\
\hline gold & 0.406 & 0.3 & 10 & 10 & 115 & 15 & 1064 \\
zinc & 0.371 & 0.3 & 10 & 10 & 105 & 10 & 1064 \\
$\mathrm{Au}-\mathrm{Zn}$ & 0.058 & 0.3 & 10 & 3 & 16.5 & 30 & 532
\end{tabular}

\section{Results and discussion}

The optical absorption spectrum of the colloidal solutions of gold nanoparticles and zinc nanoparticles produced in ethanol are shown in Fig. 1a.

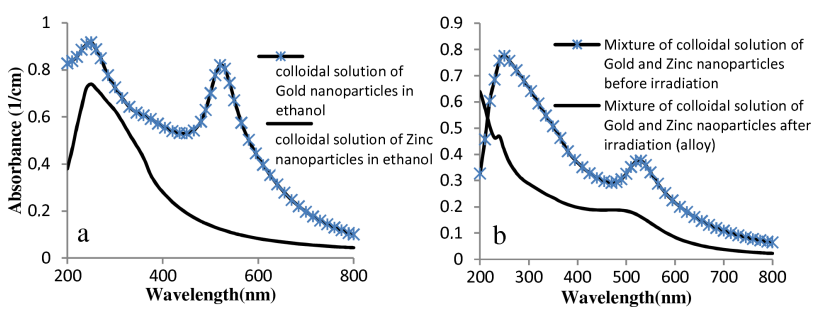

Fig. 1. (a) Optical absorption spectrum of colloidal solutions of gold nanoparticles and zinc nanoparticles in ethanol. (b) Optical absorption spectrum of mixed colloidal solutions of gold and zinc nanoparticles after and before irradiation.

The plasmon resonance absorption peak of gold nanoparticles is located at $525 \mathrm{~nm}$ and for zinc nanoparticles is at $245 \mathrm{~nm}$. The optical absorption spectrum of colloidal solutions of $\mathrm{Au}-\mathrm{Zn}$ alloy nanoparticles after irradiation by $532 \mathrm{~nm}$ wavelength for $20 \mathrm{~min}$ and the mixture of gold and zinc nanoparticles before irradiation are exhibited in Fig. 1b as well. Two distinct absorption peaks at $245 \mathrm{~nm}$ and $526 \mathrm{~nm}$ that are related to plasmon resonance of $\mathrm{Zn}$ and $\mathrm{Au}$, respectively, indicate existence of $\mathrm{Au}$ and $\mathrm{Zn}$ suspense particles in the mixture before exposing under the laser beam. Optical absorption spectrum of the mixture of colloidal nanoparticles was changed after irradiation and existence of a distinct peak at $496 \mathrm{~nm}$ that was located between plasmon resonances of gold and zinc, is the indicator of alloy formation. Also the considerable movement of the absorption peak of gold nanoparticles toward blue wavelength and intense downfall in the absorption of gold and zinc nanoparticles as well, express gold and zinc nanoparticles were removed and a new and different material was synthesized. For more accurate consideration and determination of components of colloidal solution resulting from laser irradi- ation, XRD pattern from the layered alloy sample on a silicon wafer was prepared. Figure 2 shows XRD pattern.

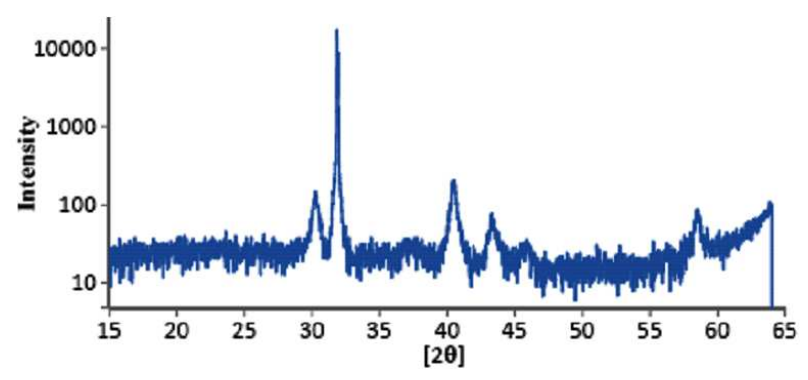

Fig. 2. XRD pattern of $\mathrm{Au}-\mathrm{Zn}$ alloy nanoparticles.

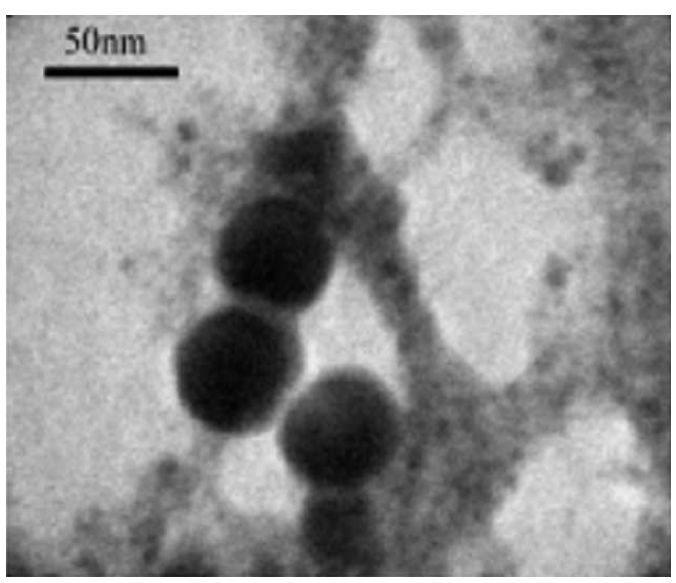

Fig. 3. TEM image of Au-Zn alloy nanoparticles.

In this pattern there are four peaks at $31.8,40.6,43.3$ and 45.8 degrees that prove formation of crystal from $\mathrm{Au}-\mathrm{Zn}$ alloy nanoparticles in colloidal solution, and its crystal composition is $\mathrm{Au}_{3} \mathrm{Zn}_{1}$. Figure 3 exhibits the image captured by TEM of $\mathrm{Au}-\mathrm{Zn}$ alloy nanoparticles. TEM image of alloy solution indicates that the procedure of alloy forming was completed following $30 \mathrm{~min}$ irradiation and spherical $\mathrm{Au}-\mathrm{Zn}$ alloy nanoparticles were synthesized. The mean diameter of particles is $18 \mathrm{~nm}$. 


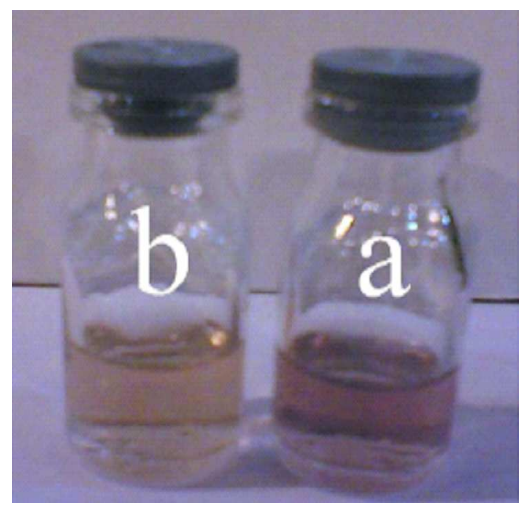

Fig. 4. The image of colloidal solution of (a) the mixture of gold and zinc nanoparticles before the irradiation and (b) the mixture of gold and zinc nanoparticles after irradiation $(\mathrm{Au}-\mathrm{Zn}$ alloy nanoparticles).

The color of the mixed colloidal solutions of gold and zinc nanoparticles in ethanol before the laser irradiation is violet but after accomplishment of irradiation by $532 \mathrm{~nm}$ wavelength, a sensible color change is occurring and the color of solution becomes orange and this occurrence is the indicator of alloy formation and synthesis of new nanoparticles. The color change of mixed solution of gold and zinc colloidal nanoparticles after and before irradiation has been shown in Fig. 4.

\section{Conclusion}

In this research colloidal nanoparticles of gold and zinc were prepared by laser ablation method in ethanol and then by irradiating of the colloidal mixture of above metal nanoparticles, $\mathrm{Au}-\mathrm{Zn}$ metal alloy nanoparticles have been produced. Plasmon absorption peak of the produced alloy nanoparticles is at $496 \mathrm{~nm}$ and TEM images express or indicate the formation of spherical particles with $18 \mathrm{~nm}$ as mean size. Also XRD pattern indicates that the main crystal structure of metal alloy is $\mathrm{Au}_{3} \mathrm{Zn}_{1}$.

\section{References}

[1] D.H. Chen, C.J. Chen, J. Mater. Chem. 12, 1557 (2002).

[2] K. Kim, K.L. Kim, S.J. Lee, Chem. Phys. Lett. 403, 77 (2005).

[3] M.J. Kim, H.J. Na, K.C. Lee, E.A. Yoo, M. Lee, J. Mater. Chem. 13, 1789 (2003).

[4] S. Link, Z.L. Wang, M.A. El-Sayed, J. Phys. Chem. B 103, 3529 (1999).

[5] F. Hajiesmaeilbaigi, A. Motamedi, Laser Phys. Lett. 3, 1 (2006).

[6] M.P. Mallin, C.J. Murphy, Nanoletters 2, 1235 (2002).

[7] M. Moskovitz, I. Srnova-Sloufova, B. Vlckova, J. Chem. Phys. 116, 10435 (2002).

[8] S. Link, M. El-Sayed, J. Phys. Chem. B 103, 8410 (1999).

[9] A.T. Izgaliev, A.V. Simakin, G.A. Shafeev, Quantum Electron. 34, 47 (2004).

[10] F. Hajiesmaeilbaigi, A. Mohamadalipour, J. Sabaghzadeh, S. Hoseinkhani, H. Fallah, Laser Phys. Lett. 3, 252 (2006).

[11] Jean-Pierre-Abid, Ph.D. Thesis, EPFL, no. 2823, 2003.

[12] E. Juárez-Ruiz, U. Pal, J.A. Lombardero-Chartuni, A. Medina, J.A. Ascencio, Appl. Phys. A, Mater. Sci. Proc. 86, 441 (2007). 